\title{
Family history of myocardial infarction as an independent risk factor for coronary heart disease
}

\author{
Y FRIEDLANDER, ${ }^{\star} †$ J D KARK,$\star \ddagger$ Y STEIN`† \\ From the $\star$ ferusalem Lipid Research Clinic and the $\nmid$ Department of Medicine B, Hadassah University Hospital; and \\ the $\ddagger$ Department of Social Medicine, Hadassah Medical Organisation and Hebrew University, Hadassah School of \\ Public Health and Community Medicine, ferusalem, Israel
}

SUMMARY The hypothesis that a family history of heart attack before the age of 60 years is an independent risk factor for coronary heart disease was examined in a random sample of 1044 men aged 40-70. Data on personal and family history, smoking, weight, height, plasma lipid and lipoprotein concentrations, blood pressure, and resting and exercise electrocardiograms were collected according to the standard Lipid Research Clinics protocol. A history of heart attack in first degree relatives was ascertained by interviewing the participants. Evidence of coronary heart disease was found in 123 men (reported heart attack in 20, electrocardiographic findings of ischaemic heart disease at rest in 40, and electrocardiographic findings during heart rate limited exercise in 63). Subjects with coronary heart disease had considerably higher concentrations of total cholesterol, higher blood pressures, and lower concentrations of high density lipoprotein cholesterol than those without. Twenty nine per cent of the subjects with coronary heart disease reported a history of heart attack in a first degree relative before 60 years of age compared with $19 \%$ of those without. In a multivariate logistic model, the coefficients for age, cholesterol concentrations, and hypertension were all positive and statistically significant. The coefficient for HDL cholesterol concentration was negative and significant. A family history of heart attack showed a significant positive association, indicating a relation with coronary heart disease that is independent of the other variables in the model. The relation persisted in apparently asymptomatic patients with coronary heart disease.

There is ample evidence that coronary heart disease tends to cluster in families. ${ }^{1-4}$ The major documented risk factors for the development of coronary heart disease have important genetic determinants..$^{5-8}$ They may account for most of the familial aggregation of coronary heart disease. ${ }^{y-11}$ The question arises whether the aggregation of coronary heart disease is due to factors other than the familial aggregation of the established coronary heart disease risk factors. A family history of heart attack or coronary heart disease was shown to be a strong predictor of coronary heart disease even after adjustment for other risk factors. ${ }^{12}$ The present report includes data from the Jerusalem Lipid Research Clinic Prevalence Study. ${ }^{13}$ The objective of the present study was to evaluate the associa-

Requests for reprints to Dr Y Friedlander, Department of Medicine B, Hadassah University Hospital, Ein Karem/Jerusalem, Israel.

Accepted for publication 30 October 1984 tion of a history of heart attack before the age of 60 years in first degree relatives with the prevalence of coronary heart disease in study participants and to test the hypothesis that this association is independent of other risk factors.

\section{Subjects and methods}

\section{STUDY POPULATION}

The basic design of the Jerusalem Lipid Research Clinic has been described in detail. ${ }^{13}$ At the preliminary examination 8646 boys and girls, aged 17 years, were interviewed at their military induction health examination. A $56 \%$ random sample of their parents were also invited to attend; these subjects formed the basis of the study group. At this visit sociodemographic information and data on the reported use of medications were collected and fasting plasma cholesterol, triglyceride, and high density lipoprotein (HDL) cholesterol concentrations were measured. Of 
the total of 6952 parents who were examined at the first visit, a random sample of $44 \%$ of the fathers and $20 \%$ of the mothers were invited for a second more extensive examination. The attendance rates were $72.0 \%$ and $79.7 \%$ at the two examinations. At the second visit detailed information on the use of medications, diet during the $\mathbf{2 4}$ hour period before examination, alcohol consumption, and cigarette smoking as well as medical and family history were recorded according to the standardised Lipid Research Clinics protocol. ${ }^{14}$ The examination included determination of plasma cholesterol, triglyceride, and lipoprotein cholesterol concentrations, including low density lipoprotein (LDL) and HDL cholesterol, blood pressure and anthropometric measurements, and a resting and exercise electrocardiogram. Blood pressure was measured four consecutive times using the conventional mercury sphygmomanometer for the first and third measurements and a zero muddler device for the second and fourth. Blood pressure was measured on the right arm with the subject seated. The systolic and the fifth phase diastolic pressures were recorded. For this analysis the mean of the two standard readings was used. Hypertension was defined as systolic blood pressure $\geqslant 140 \mathrm{~mm} \mathrm{Hg}$ or diastolic pressure $\geqslant 90 \mathrm{~mm}$ $\mathrm{Hg}$ or the use of antihypertensive medication by the subject. The Quetelet index (weight/height ${ }^{2}$ ) was used as a measure of body mass.

\section{FAMILY HISTORY}

At the second visit, data on the subjects' family health history were collected by a trained interviewer using the Lipid Research Clinics standardised questionnaire.$^{14} \mathrm{~A}$ series of questions provided information on the occurrence of myocardial infarction or angina pectoris before the age of 60 years in fathers, mothers, siblings, and children. For the present analysis a positive family history of myocardial infarction was defined as the occurrence of myocardial infarction before 60 years of age in one or more first degree relatives of the subject. The information obtained about the family health history was not validated against hospital or family doctor records.

\section{RESTING ELECTROCARDIOGRAM}

During the second visit each subject had a resting 12 lead electrocardiogram recorded in the supine position by technicians trained in the standardised performance of the procedure. Electrocardiograms were recorded on both paper and magnetic tape and sent for interpretation to the projects' central electrocardiographic laboratory in Birmingham, Alabama, USA. Electrocardiograms were read by computer as well as by two independent coders according to the Minnesota Code. ${ }^{15}$

\section{EXERCISE ELECTROCARDIOGRAM}

Submaximal exercise testing using an age specific target heart rate was performed according to the modified Bruce protocol. ${ }^{16}$ Before the exercise test a doctor took a medical history and performed a physical examination. Based on this evaluation subjects were either considered to be eligible for the treadmill test or excluded from the study. Subjects started walking on the treadmill at $1.7 \mathrm{mph}$ on a $10 \%$ gradient for the first three minutes. The speed and the slope were increased every three minutes until the subject reached $85-90 \%$ of his age adjusted predicted maximum heart rate. Tests were terminated prematurely because of fatigue or medical contraindication to continued exercise. Heart rate and blood pressure were recorded at the end of each three minute stage of exercise and at $0,2,4$, and 6 minutes after exercise. A six lead (V4, V5, V6, X, Y, and Z) electrocardiogram was recorded during and after the exercise at the same times as above.

\section{CRITERIA FOR CORONARY HEART DISEASE}

Subjects were considered to have coronary heart disease if they $(a)$ reported an admission to hospital because of a heart attack, $(b)$ had resting ischaemic electrocardiographic abnormalities (including major and minor $\mathrm{Q}$ wave abnormalities (Minnesota codes 1.1.1-1.1.7; 1.2.1-2.8; 1.3.1-3.6), major ST depression $(4.1 ; 4.2)$, and major $T$ wave abnormalities $(5.1$; 5.2 , and (c) had positive ischaemic electrocardiographic findings on the treadmill. The exercise test was defined as positive if ST segment depression during or after exercise exceeded $1 \mathrm{~mm}$ as measured by trained coders or a $10 \mathrm{mV} / \mathrm{s}$ fall in ST integral from its resting value assessed by a computer coding system. Hypotension and development of angina on the treadmill were also criteria for a positive exercise test. ${ }^{14}$

\section{STATISTICAL ANALYSIS}

Univariate and multivariate analyses were performed by a linear logistic regression model. This procedure models the disease status (that is, the prevalence of coronary heart disease) as an exponential function of a linear combination of the risk factors. Under each model the predicted probability of coronary heart disease as a function of $n$ independent variables was estimated by:

$$
P(C H D)=\exp \left(b_{o}+\sum_{i=1}^{n} b_{i} X_{i}\right) /\left(1+\exp \left(b_{o}+\sum_{i=1}^{n} b_{i} X_{i}\right)\right)
$$

The regression coefficients were computed using the Walker-Duncan maximum likelihood procedure. ${ }^{17}$ The statistical significance of coefficients in the model was tested by dividing the coefficients by their standard errors. The estimated regression coefficients for a risk factor expressed as a dummy variable or the 
Table 1 Mean (SD) plasma lipid and lipoprotein concentrations, body mass index, and prevalence of hypertension, smoking, and family history of heart attack among male subjects with and without coronary heart disease (CHD)

\begin{tabular}{lcc}
\hline & With $C H D(n=123)$ & Without $C H D(n=921)$ \\
\hline Age (yr) & $52.1(7.0)$ & $49.8(6.4)$ \\
Total cholesterol $(\mathrm{mg} / 100 \mathrm{ml})$ & $212.8(40.6)$ & $201.3(37.6)$ \\
LDL cholesterol $(\mathrm{mg} / 100 \mathrm{ml})$ & $138.8(35.2)$ & $128.6(32.5)$ \\
HDL cholesterol (mg/100 ml) & $38.8(8.3)$ & $41.0(10.4)$ \\
Triglyceride $(\mathrm{mg} / 100 \mathrm{ml})$ & $172.2(73.5)$ & $161.1(100.7)$ \\
Body mass index (weight/height $\left.{ }^{2}\right)$ & $2.68(0.39)$ & $2.63(0.34)$ \\
\% of patients: & 30.9 & 40.0 \\
Smoking & 43.9 & 27.6 \\
With hypertension & 28.5 & 19.1 \\
With family history of heart attack & & \\
\hline
\end{tabular}

LDL, low density lipoprotein; HDL, high density lipoprotein.

Conversion: traditional to SI units—cholesterol: $1 \mathrm{mg} / 100 \mathrm{ml} \approx 0.03 \mathrm{mmol} / \mathrm{l}$; triglyceride: $1 \mathrm{mg} / 100 \mathrm{ml} \approx 0.01 \mathrm{mmol} / \mathrm{l}$.

standardised coefficients for continuous variables, when exponentiated, yield an estimate of the odds ratio.

\section{Results}

The study population included 1044 men aged 40-70 years from the second visit random recall group. Of these, 20 reported a previous heart attack, 40 had ischaemic resting electrocardiogram abnormalities, and 63 developed ischaemic electrocardiographic findings during the exercise test. Table 1 summarises the characteristics of the 123 subjects with (study group) and the 921 subjects without (control group) coronary heart disease. The mean age of those subjects with coronary heart disease was two years higher than that of those without. The study group had higher mean values of total cholesterol and LDL cholesterol and lower mean values of HDL cholesterol than the control group. There was no overt difference in body mass index between the two groups. Of the study and control groups, $31 \%$ and $40 \%$ respectively were current smokers and $29 \%$ and $24 \%$ respectively were ex-smokers. The prevalence of hypertension was higher in the study group (44\%) than in the control group (28\%). A greater proportion of the study group reported a history of heart attack in a first degree relative (29\%) than of the control group (19\%).

Table 2 shows the results of logistic modelling with a set of risk factors. In the univariate analysis, age, total cholesterol and HDL cholesterol concentrations, and hypertension were statistically significant predictors of coronary heart disease. As expected, age, total cholesterol concentrations, and hypertension were positive correlates whereas HDL cholesterol concentration was inversely associated with coronary heart disease. Smoking showed an inverse association (not statistically significant) and body mass index a weak and not statistically significant correlation with disease prevalence in our sample. In the multivariate analysis the negative association with HDL cholesterol was strengthened. The multivariate coefficient for hypertension was smaller than the univariate coefficient, probably owing to the adjustment for age and body mass. The association with smoking was unchanged and that with body mass eliminated. The $\chi^{2}$ associated with the likelihood ratios was higher for the multivariate model, which included total cholesterol, than the $\chi^{2}$ for the model with LDL cholesterol. We therefore preferred the model with total cholesterol, although this did not significantly increase prediction.

Table 3 shows the logistic coefficient and the relative odds with its $95 \%$ confidence interval for family

Table 2 Logistic regression models for coronary heart disease (CHD) in 123 subjects with and 921 without coronary heart disease as a function of age, total plasma cholesterol and high density lipoprotein (HDL) cholesterol concentrations, smoking, hypertension, and body mass index

\begin{tabular}{|c|c|c|c|c|c|c|}
\hline \multirow[t]{2}{*}{ Independent variables } & \multicolumn{3}{|c|}{ Univariate model } & \multicolumn{3}{|c|}{ Multivariate model } \\
\hline & $\begin{array}{l}\text { Regression } \\
\text { coefficient }\end{array}$ & Odds ratio & CIt & $\begin{array}{l}\text { Regression } \\
\text { coefficient }\end{array}$ & Odds ratio & $\mathrm{CIt}$ \\
\hline $\begin{array}{l}\text { Age } \\
\text { Total cholesterol } \\
\text { HDL cholesterol } \\
\text { Smoking } \\
\text { Hypertension } \\
\text { Body mass index }\end{array}$ & $\begin{array}{l}0.051^{\star \star} \\
0.008^{\star \star} \\
-0.022^{\star} \\
-0.399 \\
0.721^{\star \star} \\
1.080\end{array}$ & $\begin{array}{l}1 \cdot 39 \\
1.34 \\
0.79 \\
0.67 \\
2 \cdot 06 \\
1 \cdot 14\end{array}$ & $\begin{array}{l}1.17-1.66 \\
1.12-1.61 \\
0.65-0.97 \\
0.44-1.02 \\
1.37-3.09 \\
0.95-1.37\end{array}$ & $\begin{array}{l}0.048^{\star \star} \\
0.007^{\star \star} \\
-0.032^{\star \star} \\
-0.410 \\
0.481^{\star} \\
0.064\end{array}$ & $\begin{array}{l}1.36 \\
1.32 \\
0.72 \\
0.66 \\
1.62 \\
1.02\end{array}$ & $\begin{array}{l}1.14-1.65 \\
1.09-1.59 \\
0.58-0.90 \\
0.44-1.01 \\
1.07-2.41 \\
0.84-1.25\end{array}$ \\
\hline
\end{tabular}

${ }^{\star} \mathrm{p}<0.05 ; \star \star \mathrm{p}<0.01$

$+95 \%$ confidence interval for the odds ratio. 
Table 3 Logistic regression coefficients for family history of heart attack in all 123 subjects with and 921 without coronary heart disease (CHD) and separately in 48 asymptomatic subjects with and 921 without $C H D$

\begin{tabular}{|c|c|c|c|c|c|c|}
\hline & \multicolumn{3}{|c|}{ Univariate model } & \multicolumn{3}{|c|}{ Multivariate model $\uparrow$} \\
\hline & $\begin{array}{l}\text { Regression } \\
\text { coefficient }\end{array}$ & Odds ratio & $C I \ddagger$ & $\begin{array}{l}\text { Regression } \\
\text { coefficient }\end{array}$ & Odds ratio & $C I \ddagger$ \\
\hline $\begin{array}{l}\text { Total group } \\
\text { Asymptomatic group } \$\end{array}$ & $\begin{array}{l}0.521^{\star} \\
1.020\end{array}$ & $\begin{array}{l}1 \cdot 68 \\
2 \cdot 78\end{array}$ & $\begin{array}{l}1.09-2.60 \\
1.51-5.09\end{array}$ & $\begin{array}{l}0.491^{\star} \\
1.00^{\star \star}\end{array}$ & $\begin{array}{l}1 \cdot 63 \\
2 \cdot 72\end{array}$ & $\begin{array}{l}1.06-2.52 \\
1.48-4.99\end{array}$ \\
\hline
\end{tabular}

history of heart attack as a predictor of coronary heart disease. Based on the total study group the estimated odds of coronary heart disease being present in a subject with a family history of heart attack among his first degree relatives was 1.63 times that of a subject without this hisory when all other risk factors are controlled for. The $95 \%$ confidence interval for the odds ratio ranged from 1.06 to 2.52 .

Prevalence studies may be subject to a number of well described biases. Our data allowed us to examine the possibility of bias resulting from a difference in recall of family history in subjects with and without coronary heart disease. Subjects with coronary heart disease who are aware of their condition may tend to over-report a family history of heart attack, and this could strengthen the association between family history and the disease. Subjects with asymptomatic coronary heart disease should be free of this potential bias. Thus all subjects with a history of heart attack and resting electrocardiographic abnormalities or those with a clinical picture of angina pectoris were excluded from analysis. The inclusion only of subjects with positive ischaemic electrocardiographic findings on the exercise test may produce a subgroup of coronary heart disease patients who are less likely to be aware of their medical condition. In this subgroup the positive association between family history and coronary heart diseasee was further strengthened (Table 3).

The odds ratio exceeded 2.7 both in the univariate and the multivariate model, with a $95 \%$ confidence interval ranging from 1.48 to 4.99 .

\section{Discussion}

The findings in our study that total plasma cholesterol and HDL cholesterol concentrations and hypertension are statistically significant correlates of coronary heart disease are consistent with the findings of numerous other epidemilogical studies.

In the univariate analysis, heart attack before the age of 60 in first degree relatives was a significant predictor of coronary heart disease. With multivariate analysis the family history maintained its predictive strength in the presence of the other risk factors.

The mear: ages of the subjects with and without coron: heart disease were 52 and 50 years respectivtiy, it th very few subjects aged under 40 . Consequently, nearly all the parents of our study population were over 60 years old at the time of the study. Thus all had an equal probability of developing myocardial infarction before the age of 60 . The mean number of siblings was similar in both groups (5.1 in study group and 5.0 in controls), but no data on the siblings' age and birth order were collected. We reran two logistic models, one using only parental history of myocardial infarction and the second only sibling history of myocardial infarction. The logistic coefficients were similar for both models, though they were somewhat stronger for that using sibling history. Small differences in the age distribution of siblings due to differences in the age of the study group and controls should be adequately controlled for by age adjustment in the logistic model. This assumption wuuld be incorrect, however, if birth order or family spacing were determinants of coronary heart disease. The higher odds associated with sibling history than parental history indicate an increased risk associated with a history of heart attack in first degree relatives when the condition presented early in life.

The introduction of the family history variable as the last term in the stepwise logistic regression model did not alter the coefficients for the risk factors in the model. This indicates that the risk associated with family history is independent of the other major risk factors. This does not, however, imply lack of a familial pattern in the values for total cholesterol or HDL cholesterol or blood pressure in our data. ${ }^{18}$ What this does appear to mean is that familial aggregation of these risk factors accounted for only a small part of the clustering of coronary heart disease in families. This clustering would therefore appear to reflect characteristics other than the classic risk factors that 
were measured in our study. These findings that the aggregation of coronary heart disease is not entirely explained by familial patterns in the different risk factors are consistent with other studies. ${ }^{19-21}$ Nevertheless, at least one report appeared fully to explain the clustering of coronary heart disease among relatives by the familial aggregation of the documented risk factor. ${ }^{11}$ We are unable to assess the relative importance of heredity and environmental factors in the association of family history with coronary heart disease. The contribution of heredity to the familial aggregation of ischaemic heart disease may be dominant. ${ }^{22}$ Nevertheless, environmental factors such as diet (independent of lipid concentrations) and psychosocial factors that are shared by family members could contribute independently or interact with the heredity factor.

Several possible problems in our study need to be considered. Firstly, there was no verification of the reported family history, and therefore the validity of these data cannot be confirmed. Other studies such as the Tecumseh Community Health Study and the Tromso Heart Study reported satisfactory validity of reported family history of heart attack. ${ }^{19} 23$ There are no published data in Israel relating to this question. Secondly with prevalence data the possibility exists of bias due to factors such as selective survival, change in behaviour, and recall bias. For instance, in this study the proportion of current smokers among the study group was lower (31\%) than among the control group $(40 \%)$, although smoking has been shown to be a risk factor for myocardial infarction in Israeli men in a large prospective study. ${ }^{24}$ Selective survival and recall bias could affect the association of family history with coronary heart disease. In particular, subjects with coronary heart disease may over-report a family history of heart attack. Biased recall, however, is unlikely to be responsible for our family history findings. Restricting the analysis to (probably) asymptomatic subjects did not weaken the association. The exercise electrocardiogram in asymptomatic subjects is known to be of relatively low specificity and small predictive value. Such misclassification would tend to reduce the odds ratic estimates towards the null value assuming that there is no differential misclassification of subjects with and without a positive family history. Thus our findings of a statistically significant association of coronary heart disease with a positive family history is probably an underestimate of the true association.

Our data suggest that a family history of myocardial infarction may be an important independent risk factor for the development of coronary heart disease in Israeli men. Further studies are needed to substantiate this finding and to explore the nature of this familial risk factor and the mechanism by which it pro- duces coronary heart disease in our population.

This study was supported by the US National Heart, Lung, and Blood Institute.

\section{References}

1 Rose G. Familial patterns in ischaemic heart disease. $\mathrm{Br}$ f Preo Soc Med 1964; 18: 75-80.

2 Slack J, Evans KA. The increased risk of death from ischaemic heart disease in first degree relatives of 121 men and 96 women with ischaemic heart disease. $\mathcal{F}$ Med Genet 1966; 3: 239-57.

3 Phillips RL, Lilienfeld AM, Diamond EL, Kagan A. Frequency of coronary heart disease and cerebovascular accidents in patients and sons of coronary heart disease index cases and controls. Am $\mathcal{F}$ Epidemiol 1974; 100: 87100.

4 Rissanen AM. Familial aggregation of coronary heart disease in a high incidence area (North Karelia, Finland). Br Hean F 1979; 42: 294-303.

5 Sing CF, Orr JD. Analysis of genetic and environmental sources of variation in serum cholesterol in Tecumseh, Michigan. IV Separation of polygene from common environment effects. Am f Hum Genet 1978; 30: 491-504.

6 Rao DC, Morton NE, Gulbrandsen CL, Rhoads GG, Kagan A, Yee S. Cultural and biological determinants of lipoprotein concentrations. Ann Hum Genet 1979; 42: 467-77.

7 Morton NE, Gulbrandsen CL, Rao DC, Rhoads GG, Kagan A. Determinants of blood pressure in JapaneseAmerican families. Hum Genet 1980; 53: 261-6.

8 Rotter JI. Genetic heterogeneity within diabetes mellitus, a review. In: Sing CF, Skolnick M, eds. Genetic analysis of common diseases: applications to predictive factors in coronary disease. New York: Alan R Liss, 1979: 135-55.

9 Deutscher S, Epstein FH, Keller JB. Relationships between familial aggregation of coronary heart disease and risk factors in the general population. Am $\mathcal{Y}$ Epidemiol 1969; 89: 510-20.

10 Aro A. Serum lipids and lipoproteins in first degree relatives of young survivors of myocardial infarction. Acta Med Scand [Suppl] 1973; 553: 7-103.

11 Rissanen AM, Nikkili EA. Aggregation of coronary risk factors in families of men with fatal and non-fatal coronary heart disease. Br Heart f 1979; 42: 373-80.

12 Sholtz RI, Rosenman RH, Brand RJ. The relationship of reported parental history to the incidence of coronary heart disease in the Western Collaborative Group Study. Am I Epidemiol 1975; 102: 350-6.

13 Slater PE, Friedlander Y, Baras M, et al. The Jerusalem Lipid Research Clinic: sampling, response and selected methodological issues. Isr I Med Sci 1982; 18: 1106-12.

14 Central Patient Registry and Coordinatory Center of the Lipid Research Clinics: Reference Manual for the Lipid Research Clinics Program Prevalence Study, Vol 1 and 2. Department of Biostatistics, University of North Carolina at Chapel Hill, Chapel Hill, NC, 1974.

15 Rose GA, Blackburn H. Cardiovascular survey methods. 
WHO Monogr Ser 1968; 56: 1-188.

16 Sheffield LT, Holt JH, Reeves TJ. Exercise graded by heart rate in electrocardiographic testing for angina pectoris. Circulation 1965; 32: 622-9.

17 Walker SH, Duncan DB. Estimation of the probability of an event as a function of several independent variables. Biometrika 1967; 54: 167-79.

18 Friedlander $Y$, Cohen $T$, Stenhouse $N$, Davies AM, Stein Y. Familial aggregation of total cholesterol, triglyceride and high-density lipoprotein-cholesterol in an Israeli population sample. Isr f Med Sci 1982; 18: 113743.

19 Forde OH, Thelle DS. The Tromso heart study: risk factors for coronary heart disease related to the occurrence of myocardial infarction in first degree relatives. Am $\mathcal{F}$ Epidemiol 1977; 105: 192-9.

20 Snowden CB, McNamara PM, Garrison RJ, Feinleib M, Kannel WB, Epstein FH. Predicting coronary heart dis- ease in siblings a multivariate assessment. The Framingham heart study. Am $\mathcal{F}$ Epidemiol 1982; 115: 217-22.

21 ten Kate LP, Boman H, Daiger SP, Motulsky AG. Familial aggregation of coronary heart disease and its relation to known genetic risk factors. Am $\mathcal{F}$ Cardiol 1982; 50: 945-53.

22 Nora JJ, Lortscher RH, Spangler RD, Nora AH, Kimberling WJ. Genetic-epidemiologic study of early-onset ischemic heart disease. Circulation 1980; 61: 503-8.

23 Napier JA, Metzner H, Johnson BC. Limitations of morbidity and mortality data obtained from family histories a report from the Tecumseh Community Health Study. Am $\mathcal{F}$ Public Health 1972; 62: 30-5.

24 Goldbourt U, Medalie JH, Neufeld HN. Clinical myocardial infarction over a five-year period. III. A multivariate analysis of incidence, the Israel Ischemic Heart Disease Study. $f$ Chronic Dis 1975; 28: 217-37. 